\title{
On trade in bilateral oligopolies with altruistic and spiteful agents
}

\author{
M. Lombardi ${ }^{1,2} \cdot$ S. Tonin ${ }^{3}$ (D
}

Received: 20 January 2019 / Accepted: 4 September 2019 / Published online: 14 September 2019 (c) The Author(s) 2019

\begin{abstract}
This paper studies the effects of altruism and spitefulness in a two-sided market in which agents behave strategically and trade according to the Shapley-Shubik mechanism. By assuming that altruistic agents have concerns for others on the opposite side of the market, it shows that agents always find advantageous to trade. However, they prefer to stay out of the market and consume their endowments when there are altruistic agents who have concerns for the welfare of those on the same side of the market, or when there are spiteful agents. These non-trade situations occur either because the necessary first-order conditions for optimality are violated or because agents' payoff functions are not concave.
\end{abstract}

Keywords Bilateral oligopoly · Noncooperative oligopoly · Nash equilibrium · Altruism and spitefulness

JEL Classification D43 - D51

\section{Introduction}

We often incorporate the preferences of others in our decision making. We do so because we intrinsically care about the welfare of other agents in the economy. In

\footnotetext{
We would like to thank Sayantan Ghosal, Antonio Villanacci, and two anonymous referees for their comments and suggestions. The usual disclaimer applies.

$凶 \quad$ S. Tonin

simone.tonin@durham.ac.uk

M. Lombardi

michele.lombardi@glasgow.ac.uk

1 Adam Smith Business School, University of Glasgow, Glasgow G12 8QQ, UK

2 Department of Economics and Statistics, University of Naples Federico II, Naples 81026, Italy

3 Durham University Business School, Durham University, Durham DH1 3LB, UK
} 
this paper, we continue a line of inquiry begun by Dubey and Shubik (1985) and Dufwenberg et al. (2011) by investigating how altruism and spitefulness influence equilibrium outcomes in imperfectly competitive markets.

We confine ourselves to a class of strategic market games introduced by Gabszewicz and Michel (1997), known as bilateral oligopoly. ${ }^{1}$ In this two-sided market model, agents act strategically and trade according to Shapley and Shubik (1977)'s mechanism (henceforth, Shapley-Shubik mechanism): they submit bids and offers to the mechanism and the price is determined by the ratio of total bids to total offers.

The cornerstone of bilateral oligopoly is the assumption that individual agents' behaviors are solely motivated by their personal concern. However, there is a considerable amount of both experimental and empirical evidence that individuals do not have independent preferences, in the sense that considerations of others influence individual behavior. This paper departs from the traditional assumption of independent preferences by assuming that agents act by considering both personal concern and concerns for the welfare of others.

By following the growing literature on behavioral economics that constructs theoretical models with altruistic/spiteful agents (e.g., Levine 1998; Graziano et al. 2017; Bourlès et al. 2017), this paper also assumes that an agent, who has a concern for others, has an overall utility function which encompasses both his internal utility (that is, a classical utility function defined over his consumption set) and internal utilities of others weighted by preference parameters. ${ }^{2}$ Each parameter reflects the degree of importance that an agent puts on the welfare of another: positive under altruism, negative under spitefulness, and zero under the classic assumption of independent preferences. We refer to this utility function as Edgeworth utility function (Edgeworth 1881).

Dufwenberg et al. (2011) show that concern for others does not affect equilibrium outcomes when markets are perfectly competitive and agents' preferences are represented by Edgeworth utility functions. In particular, they find that agents behave, as if they had classical independent preferences at competitive equilibria. Dubey and Shubik (1985) reach exactly the same conclusion with a continuum of agents in a strategic market game. By contrast, we find that altruism and spitefulness affect the volume of trade in bilateral oligopolies and, more interestingly, that this type of preferences may shrink the volume of trade down to zero. This holds even when trade produces high internal utility gains - a requirement introduced by Bloch and Ferrer (2001) in response to the non-trade situations studied by Cordella and Gabszewicz (1998). These findings are not in line with the conclusions reached in auction settings (e.g., Levine 1998; Sobel 2010), according to which concern for others does not affect equilibrium outcomes.

When agents have altruistic concerns for others on the opposite side of the market, we show that a trade (Nash) equilibrium exists under assumptions that are common in the bilateral oligopoly literature with independent preferences. As Bloch and Ferrer (2001), we also make restrictions on marginal utilities to consider economies where

\footnotetext{
1 This basic model of trade has been also studied by Bloch and Ghosal (1997), Bloch and Ferrer (2001), Dickson and Hartley (2008), Amir and Bloch (2009), among others.

2 For excellent surveys see, for instance, Fehr and Gachter (2000) and Sobel (2005).
} 
some agents have high internal gains from trade. ${ }^{3}$ This positive result can be explained as follows. When agents have independent preferences and there are high gains from trade, there exists a trade equilibrium. A property of the Shapley-Shubik mechanism is that an offer by an agent produces internal utility gains for others on the opposite side of the market. This translates, ceteris paribus, into utility gains for agents who have altruistic concerns for others on the opposite side of the market. Therefore, this type of altruism strengthens the incentives to trade and does not upset the conclusions drawn under the assumption of independent preferences. Our new proof of existence can be used to generalize Bloch and Ferrer (2001)'s existence result.

However, we also show that a trade equilibrium does not exist when agents have altruistic concerns for others on the same side of the market. We provide an example of bilateral oligopoly satisfying the classical assumptions on utility functions, as well as the assumption of high internal gains from trade, for which the non-trade equilibrium is the unique equilibrium. The economic intuition for this negative result is as follows. A property of the Shapley-Shubik mechanism is that an offer by an agent produces internal utility losses for others on the same side of the market. This translates, ceteris paribus, into a utility loss for a supplier who intends to maximize the welfare of others on the same side of the market. When such a loss is not compensated by a gain in his internal utility, the altruistic supplier would prefer to offer nothing to the market. We further clarify this point by providing a necessary condition for the existence of trade equilibria. Heuristically, this condition requires that internal utility gains from trade more than compensate for losses suffered by others on the same side of the market. This result is in line with the previous literature on altruism which shows that paradoxical situations arise when agents are too benevolent (e.g., Bergstrom 1989).

While there are many settings where agents are altruistic, there are also situations in which agents aim to outdo other agents to improve their own standing. For this reason, we also study bilateral oligopolies with spiteful agents, who are interested in minimizing the welfare of others as well as in maximizing their internal utilities. In this setting, we provide examples of bilateral oligopolies, satisfying the classical assumptions on utility functions, as well as the assumption of high internal gains from trade, for which the non-trade equilibrium is the unique equilibrium. In particular, we explore the effects of spiteful concerns both for agents on the opposite side of the market and for those on the same side of the market. Although spitefulness is detrimental to the existence of trade equilibria, we find that these negative effects are caused by two distinct factors.

In a setting where agents aim to minimize the welfare of others on the opposite side of the market, the non-existence of trade equilibria stems from the fact that in the Shapley-Shubik mechanism an offer by an agent produces internal utility gains for others on the opposite side of the market. This translates, ceteris paribus, into a utility loss for a supplier who intends to minimize the welfare of others on the opposite of the market. When such a loss is not compensated by a gain in his internal utility, a spiteful supplier would prefer to offer nothing to the market. By contrast, in a setting

\footnotetext{
${ }^{3}$ A non-trade equilibrium, also called trivial equilibrium, always exists. To study trade equilibria, Bloch and Ferrer (2001) introduce an assumption on the marginal utilities of all agents, similar to the Inada conditions, which implies high internal gains from trade.
} 
where agents aim to minimize the welfare of others on the same side of the market, we report that the non-existence is due to the non-concavity of the payoff functions.

Section 2 describes the theoretical framework and outlines the bilateral oligopoly model, with results presented in Sect. 3. Section 4 concludes. The appendix contains the proof of existence.

\section{Mathematical model}

We consider exchange economies with two types of agents, labeled 1 and 2, and two (perfectly divisible) commodities, labeled $x$ and $y$. The set of agents is $I=I^{1} \cup I^{2}$, where $I^{t}$ is the finite set of agents of type $t=1,2$. An agent $i$ is of type 1 when he is endowed with $x_{i}^{0}>0$ units of commodity $x$, but no unit of commodity $y$. Similarly, an agent $i$ is of type 2 when he is endowed with $y_{i}^{0}>0$ units of commodity $y$ but no unit of commodity $x$. Therefore, agents of different types are on different sides of the market, as they hold different commodities. We make the following assumption throughout the paper.

Assumption 1 There are at least two agents for each type.

Agent $i$ 's bundle $\left(x_{i}, y_{i}\right)$ is a (non-negative) two-dimensional vector describing how much of each commodity he consumes. An allocation $(x, y)=\left(x_{i}, y_{i}\right)_{i \in I}$ is a list of bundles. Each agent $i$ maximizes the utility function:

$$
V_{i}(x, y)=u_{i}\left(x_{i}, y_{i}\right)+\sum_{j \neq i} \gamma_{i}^{j} u_{j}\left(x_{j}, y_{j}\right)
$$

where $-1 \leq \gamma_{i}^{j} \leq 1$ for each agent $j \neq i{ }^{4}$ Agent $i$ 's utility function embodies a private and a social component. The private component is represented by the internal utility $u_{i}$ that depends on commodities that go directly to him. The social component is instead represented by the weighted sum of the internal utilities of other agents. Each parameter $\gamma_{i}^{j}$ is the weight that agent $i$ put on agent $j$ 's utility, positive under altruism, negative under spitefulness, and zero under independent preferences. Let $\gamma_{i}=\left(\gamma_{i}^{j}\right)_{j \in I}$, with $\gamma_{i}^{i}=1$, denote agent $i$ 's preference parameters. The following classical assumption on internal utilities is made.

Assumption 2 For each agent $i \in I$, the internal utility $u_{i}$ is continuous, strictly increasing and concave on $\mathbb{R}_{+}^{2}$. Furthermore, $u_{i}$ is continuously differentiable on $\mathbb{R}_{++}^{2} \cdot^{5}$

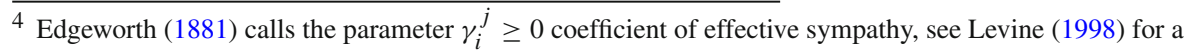
discussion on the different interpretations of $\gamma_{i}^{j}$.

5 We assume continuous differentiability only on $\mathbb{R}_{++}^{2}$ to allow the case of infinite partial derivatives along the boundary of the consumption set (see Kreps (2012), p. 58).
} 
By following the approach developed by Bloch and Ferrer (2001), we consider bilateral oligopolies in which there are high internal gains from trade, i.e., high gains from trade with respect to internal utility functions. ${ }^{6}$ This is captured by the following.

Assumption 3 There exists an agent $i$ endowed with an additively separable utility function $u_{i}\left(x_{i}, y_{i}\right)=u_{i}^{1}\left(x_{i}\right)+u_{i}^{2}\left(y_{i}\right)$ satisfying the following property: $\frac{d u_{i}^{2}(0)}{d y_{i}}=\infty$, if $i \in I^{1}$, and $\frac{d u_{i}^{1}(0)}{d x_{i}}=\infty$, otherwise.

Since the set of agents will remain fixed, an exchange economy with altruistic/spiteful agents is denoted by $(u, \gamma, w)$, where $u=\left(u_{i}\right)_{i \in I}$ is the profile of internal utilities, $\gamma=\left(\gamma_{i}\right)_{i \in I}$ is the profile of agents' preference parameters, and $w=\left(x_{i}^{0}, y_{i}^{0}\right)_{i \in I}$ is the endowment profile. An exchange economy in which agents have independent preferences is simply denoted by $(u, w)$.

In our model, agents behave strategically: each agent offers a quantity of his endowment to the market. The strategy spaces are thus given by

$$
\begin{aligned}
& S_{i}=\left\{a_{i}: 0 \leq a_{i} \leq x_{i}^{0}\right\}, \text { for each } i \in I^{1}, \\
& S_{i}=\left\{b_{i}: 0 \leq b_{i} \leq y_{i}^{0}\right\}, \text { for each } i \in I^{2} .
\end{aligned}
$$

We write $(a, b)$ for the profile of offers $\left(\left(a_{i}\right)_{i \in I^{1}},\left(b_{i}\right)_{i \in I^{2}}\right)$ and $S$ for $\prod_{i \in I} S_{i}$. Clearly, $(a, b)$ is an element of $S$. In addition, $\left(a_{-i}, b\right)$ is an element of $\prod_{j \neq i} S_{j}$, with $i \in I^{1}$, and $\left(a, b_{-i}\right)$ is an element of $\prod_{j \neq i} S_{j}$, with $i \in I^{2}$. For any profile of offers $(a, b) \in S$, the bundles assigned to agents are given by the following allocation rule:

$$
\begin{aligned}
& \left(x_{i}(a, b), y_{i}(a, b)\right)=\left(x_{i}^{0}-a_{i}, a_{i} \frac{B}{A}\right), \text { for each } i \in I^{1}, \\
& \left(x_{i}(a, b), y_{i}(a, b)\right)=\left(b_{i} \frac{A}{B}, y_{i}^{0}-b_{i}\right), \text { for each } i \in I^{2},
\end{aligned}
$$

if $A=\sum_{i \in I^{1}} a_{i}>0$ and $B=\sum_{i \in I^{2}} b_{i}>0$. Otherwise, each agent consumes only his own endowment. The ratio $\frac{B}{A}$ is the price of commodity $x$, whereas the price of $y$ is normalized to 1 . The allocation generated by $(a, b)$ is denoted by $(x(a, b), y(a, b))$.

The above allocation rule combined with $(S, u, \gamma)$ defines a bilateral oligopoly with altruistic and spiteful agents, which is denoted by $\Gamma(\gamma)$. We write $\Gamma$ to denote a bilateral oligopoly where agents have independent preferences. We adopt the following solution concept of (Nash) equilibrium.

Definition 1 An equilibrium for $\Gamma(\gamma)$ is a profile of offers $(\hat{a}, \hat{b})$ such that: for each agent $i \in I^{1}, \hat{a}_{i} \in S_{i}$ maximizes

$$
V_{i}\left(x\left(\left(a_{i}, \hat{a}_{-i}\right), \hat{b}\right), y\left(\left(a_{i}, \hat{a}_{-i}\right), \hat{b}\right)\right)
$$

\footnotetext{
6 Bloch and Ferrer (2001) assume that the restriction on marginal utilities holds for both commodities and for all agents.
} 
and for each agent $i \in I^{2}, \hat{b}_{i} \in S_{i}$ maximizes

$$
V_{i}\left(x\left(\hat{a},\left(b_{i}, \hat{b}_{-i}\right)\right), y\left(\hat{a},\left(b_{i}, \hat{b}_{-i}\right)\right)\right) .
$$

Let $(\hat{a}, \hat{b})$ be an equilibrium for $\Gamma(\gamma)$. The profile $(\hat{a}, \hat{b})$ is a non-trade equilibrium for $\Gamma(\gamma)$ if $\hat{A}=0$ or $\hat{B}=0$. The non-trade equilibrium always exists. The profile $(\hat{a}, \hat{b})$ is a trade equilibrium for $\Gamma(\gamma)$ if $\hat{A}>0$ and $\hat{B}>0$.

\section{Altruistic agents}

We start our analysis by considering altruistic agents who are characterized by positive preference parameters. They aim to maximize their internal utilities as well as the welfare of other agents.

Let us start considering the case, where agents have altruistic concerns for others on the opposite side of the market. In such a context, a trade equilibrium always exists as shown by the following theorem.

Theorem Let $(u, \gamma, w)$ be an exchange economy satisfying Assumptions 1-3. For each agent $i \in I^{t}$, let $\gamma_{i}$ be such that $\gamma_{i}^{j}=0$ for each $j \in I^{t} \backslash\{i\}$ and $\gamma_{i}^{j} \geq 0$ for each $j \in I \backslash I^{t}$, for each $t=1,2$. Then, there exists a trade equilibrium for $\Gamma(\gamma)$.

The proof can be found in the appendix and it adopts techniques which are similar to the ones used by Dubey and Shubik (1978) and Bloch and Ferrer (2001). Roughly speaking, the proof consists of the following steps. First, we show the existence of an equilibrium in a perturbed bilateral oligopoly using the Kakutani fixed point theorem, which requires convex-valued best response correspondences. This requirement is met whenever agents' utility functions are concave. For this reason, we restrict our analysis to altruistic agents having Edgeworth utility functions, which are concave, because they are sum of concave functions. Alternatively, convex-valued best response correspondences are guaranteed by assuming concavity over allocations. However, this is a very stringent assumption (on this point, see Dufwenberg et al. 2011). Second, using the Uniform Monotonicity Lemma of Dubey and Shubik (1978), we show that equilibrium prices of each perturbed bilateral oligopoly are bounded away from zero and from above. Note that this result cannot be obtained when agents have altruistic concerns for others on the same side of the market. ${ }^{7}$ Third, we show that the equilibrium strategy of the agent satisfying Assumption 3 is bounded away from zero in each perturbed bilateral oligopoly. This result is inspired by Bloch and Ferrer (2001) and the main novelty is the way in which the Kuhn-Tucker theorem is used. Finally, we show the convergence of the sequence of perturbed equilibria to a trade equilibrium of $\Gamma(\gamma)$ when the perturbation goes to zero.

As a corollary, we obtain the following generalization of the existence result of Bloch and Ferrer (2001) for bilateral oligopolies with independent preferences.

\footnotetext{
7 The reason is that an offer by this type of altruistic agents produces internal utility losses for others on the same side of the market-in other words, inequality (4) in the proof of Lemma 2 no longer holds.
} 
Corollary Let $(u, w)$ be an exchange economy satisfying Assumptions $1-3$. Then, there exists a trade equilibrium for $\Gamma$.

The proof follows immediately from the theorem when $\gamma_{i}$ is set equal to zero for all agents. It is worth emphasizing that trade equilibria of a bilateral oligopoly with altruistic agents are different from the trade equilibria of the corresponding bilateral oligopoly with agents having independent preferences.

We now turn to the case where agents have altruistic concerns for others on the same side of the market. In sharp contrast to the previous result, we show, by means of an example, that a trade equilibrium may fail to exist, though the exchange economy satisfies Assumptions 1-3. This non-existence result is due to the negativity of the necessary first-order conditions for optimality.

Example 1 Consider an exchange economy with four agents having the following utility functions and endowments:

$$
\begin{aligned}
& V_{1}(x, y)=\sqrt{x_{1}}+y_{1}+\frac{1}{2}\left(\sqrt{x_{2}}+2 y_{2}\right) \text { and }\left(x_{1}^{0}, y_{1}^{0}\right)=(4,0), \\
& V_{2}(x, y)=\sqrt{x_{2}}+2 y_{2}+\frac{1}{2}\left(\sqrt{x_{1}}+y_{1}\right) \text { and }\left(x_{2}^{0}, y_{2}^{0}\right)=(4,0), \\
& V_{3}(x, y)=\sqrt{x_{3}}+y_{3}+\frac{1}{2}\left(\sqrt{x_{4}}+y_{4}\right) \text { and }\left(x_{3}^{0}, y_{3}^{0}\right)=(0,4), \\
& V_{4}(x, y)=\sqrt{x_{4}}+y_{4}+\frac{1}{2}\left(\sqrt{x_{3}}+y_{3}\right) \text { and }\left(x_{4}^{0}, y_{4}^{0}\right)=(0,4) .
\end{aligned}
$$

Note that the exchange economy satisfies Assumptions 1-3. By checking the necessary first-order conditions for optimality, it is straightforward to verify that the non-trade equilibrium is the unique equilibrium of the $\Gamma(\gamma)$ associated with the aforesaid exchange economy.

The reason behind this non-existence result can be explained by focusing on agent 1 's utility function. From the allocation rule (1), the final quantity of the commodity $y$ assigned to agent 2 depends negatively on the quantity of $x$ offered by agent 1 . Therefore, if agent 1's gain from consuming additional units of commodity $y$ does not outweigh his loss from a decrease in agent 2's consumption of $y$, then agent 1 maximizes his payoff by reducing his offer $a_{1}$ to zero. This can be seen by considering the derivative of the payoff function of agent 1 with respect to his offer $a_{1}$, which can be stated as follows:

$$
\frac{\partial V_{1}}{\partial a_{1}}=-\frac{\partial u_{1}}{\partial x_{1}}+\frac{\partial u_{1}}{\partial y_{1}} \frac{B}{A^{2}} a_{2}-\gamma_{1}^{2}\left(\frac{\partial u_{2}}{\partial y_{2}} \frac{B}{A^{2}} a_{2}\right) .
$$

By substituting the marginal utilities of agents 1 and 2 as well as agent 1 's preference parameter $\gamma_{1}^{2}=\frac{1}{2}$ of Example 1, one can easily verify that the above derivative is negative and then agent 1 's best strategy is $a_{1}=0$. It is well know that in the bilateral oligopoly model, we have a trade equilibrium only when there are two agents offering 
each commodity. ${ }^{8}$ Since in our example, agent 1 's best offer is always nil, the non-trade equilibrium is the unique equilibrium.

Heuristically, the non-existence result is due to the fact that agent 1 is too altruistic. This is a well-known problem in the literature of altruism where, in extreme cases, any reasonable connection between an agent's utility level and his own consumption is lost. ${ }^{9}$ The next proposition further clarifies this point by providing a necessary condition on marginal utilities as well as preference parameters for the existence of a trade equilibrium. We state this necessary condition only for the case where agents of type 1 have altruistic concerns for others on the same side of the market.

Proposition 1 Let $(u, \gamma, w)$ be an exchange economy satisfying Assumptions 1-3. For each agent $i \in I^{1}$, let $\gamma_{i}$ be such that $\gamma_{i}^{j} \geq 0$ for each $j \in I^{1} \backslash\{i\}$ and $\gamma_{i}^{j}=0$ for each $j \in I^{2}$. For each agent $i \in I^{2}$, let $\gamma_{i}^{j}=0$ for each $j \neq i$. Then, if $(\hat{a}, \hat{b})$ is a trade equilibrium, then

$$
\left.\frac{\partial u_{i}}{\partial y_{i}}\right|_{(\hat{a}, \hat{b})} \geq\left.\gamma_{i}^{j} \frac{\partial u_{j}}{\partial y_{j}}\right|_{(\hat{a}, \hat{b})}
$$

for some agents $i, j \in I^{1}$, with $i \neq j$.

The necessary condition imposes a restriction on the effects that concerns for others have on an altruistic agent's overall utility. It is worth noting that this condition is similar in spirit to a restriction introduced by Bourlès et al. (2017) in a framework where exchange takes place over a network structure. As already noted above, this necessary condition is not a sufficient one, because equilibrium prices in the perturbed bilateral oligopoly may be not bounded away from zero when agents have altruistic concerns for others on the same side of the market (see footnote 7).

\section{Spiteful agents}

We now consider bilateral oligopolies with spiteful agents. Such agents are characterized by negative preference parameters and they aim to minimize the welfare of other agents in the economy. Although there are high internal gains from trade, we show, by means of examples, that a trade equilibrium may fail to exist when there are spiteful agents.

We first turn to the case where agents have spiteful concerns for others on the opposite side of the market. In such a case, the non-existence of a trade equilibrium is due to the negativity of the necessary first-order conditions for optimality.

\footnotetext{
8 When $a_{1}=0$, the allocation rule (1) implies that agent 2 can increase his utility by decreasing his offers of commodity $x$, because for any $a_{2}$, he gets all the amount $B$ of commodity $y$ offered in the market. In such a case, the agent 2's payoff function is not continuous on $S_{2}$ and he does not have a best strategy.

9 See Ythier (2006) for an extensive discussion of the problem as well as the restrictions suggested by the literature to rule it out.
} 
Example 2 Consider an exchange economy with four agents having the following utility functions and endowments:

$$
\begin{aligned}
V_{i}(x, y)= & \sqrt{x_{i}}+y_{i}-\frac{1}{2}\left(\sqrt{x_{3}}+y_{3}\right)-\frac{1}{2}\left(\sqrt{x_{4}}+y_{4}\right) \text { and }\left(x_{i}^{0}, y_{i}^{0}\right)=(4,0), \\
& \text { for } i=1,2 \\
V_{i}(x, y)= & \sqrt{x_{i}}+y_{i}-\frac{1}{2}\left(\sqrt{x_{1}}+y_{1}\right)-\frac{1}{2}\left(\sqrt{x_{2}}+y_{2}\right) \text { and }\left(x_{i}^{0}, y_{i}^{0}\right)=(0,4), \\
& \text { for } i=3,4 .
\end{aligned}
$$

Note that the exchange economy satisfies Assumptions 1-3. It is possible to verify that the non-trade equilibrium is the unique equilibrium of the $\Gamma(\gamma)$ associated with the aforesaid exchange economy. ${ }^{10}$

The intuition behind this non-existence result can be explained by focusing on agent 1 's utility function. From the allocation rule (2), the final quantities of commodity $x$ assigned to agents 3 and 4 depend positively on the quantity $x$ offered by agents 1 and 2. Therefore, if agent 1's gain from consuming additional units of commodity $y$ does not outweigh his loss from an increase in agents 3 and 4' consumption of commodity $x$, then agent 1 may maximize his payoff by reducing his offer $a_{1}$ to zero. This can also be seen by considering the derivative of the payoff function of agent 1 with respect to his offer, which can stated as follows:

$$
\frac{\partial V_{1}}{\partial a_{1}}=-\frac{\partial u_{1}}{\partial x_{1}}+\frac{\partial u_{1}}{\partial y_{1}} \frac{B}{A^{2}} a_{2}+\gamma_{1}^{3}\left(\frac{\partial u_{3}}{\partial x_{3}} \frac{b_{3}}{B}\right)+\gamma_{1}^{4}\left(\frac{\partial u_{4}}{\partial x_{4}} \frac{b_{4}}{B}\right)
$$

Heuristically, if the absolute values of $\gamma_{1}^{3}$ and $\gamma_{1}^{4}$ are high enough, then agent 1 's best strategy is $a_{1}=0 .{ }^{11}$ The same argument applies to other agents. We thus conclude that there is no strategy profile that can satisfy the necessary first-order conditions for optimality, and therefore, the non-trade equilibrium is the unique equilibrium of $\Gamma(\gamma)$.

We finally turn to the case where agents have spiteful concerns for others on the same side of the market. In such a case, the non-existence of a trade equilibrium is due to the non-concavity of payoff functions.

\footnotetext{
10 This can be verified by solving the necessary first-order condition for optimality with any computer algebra system.

11 Recall that $\gamma_{i}^{j}<0$ under spitefulness.
} 
Example 3 Consider an exchange economy with four agents having the following utility functions and endowments:

$$
\begin{aligned}
& V_{1}(x, y)=\frac{2}{3} x_{1}+y_{1}-\frac{1}{2}\left(\sqrt{x_{2}}-\left(\frac{1}{100}+y_{2}\right)^{-2}\right) \text { and }\left(x_{1}^{0}, y_{1}^{0}\right)=(4,0), \\
& V_{2}(x, y)=\sqrt{x_{2}}-\left(\frac{1}{100}+y_{2}\right)^{-2} \text { and }\left(x_{2}^{0}, y_{2}^{0}\right)=(4,0) \\
& V_{i}(x, y)=\sqrt{x_{i}}+y_{i} \text { and }\left(x_{i}^{0}, y_{i}^{0}\right)=(0,4), \text { for } i=3,4 .
\end{aligned}
$$

Note that the exchange economy satisfies Assumptions 1-3. In addition, note that only agent 1 has spiteful concern for agent 2 . It is possible to verify that the nontrade equilibrium is the unique equilibrium of the $\Gamma(\gamma)$ associated with the aforesaid exchange economy.

In the example, the strategy profile $\left(\hat{a}_{1}, \hat{a}_{2}, \hat{b}_{3}, \hat{b}_{4}\right)=(2.46,3.60,0.44,0.44)$ is the unique solution to the necessary first-order conditions for optimality of all agents. ${ }^{12}$ However, this profile does not correspond to an equilibrium, because agent 1 can find a unilateral profitable deviation. Indeed, the figure below shows the shape of agent 1 's payoff function when other agents offer $\left(\hat{a}_{2}, \hat{b}_{3}, \hat{b}_{4}\right)$. It is immediate to see that agent 1 's payoff function is convex and $\hat{a}_{1}$ corresponds to a minimum point. Therefore, $\left(\hat{a}_{1}, \hat{a}_{2}, \hat{b}_{3}, \hat{b}_{4}\right)$ cannot be an equilibrium and so the non-trade equilibrium is the unique equilibrium of $\Gamma(\gamma)$.

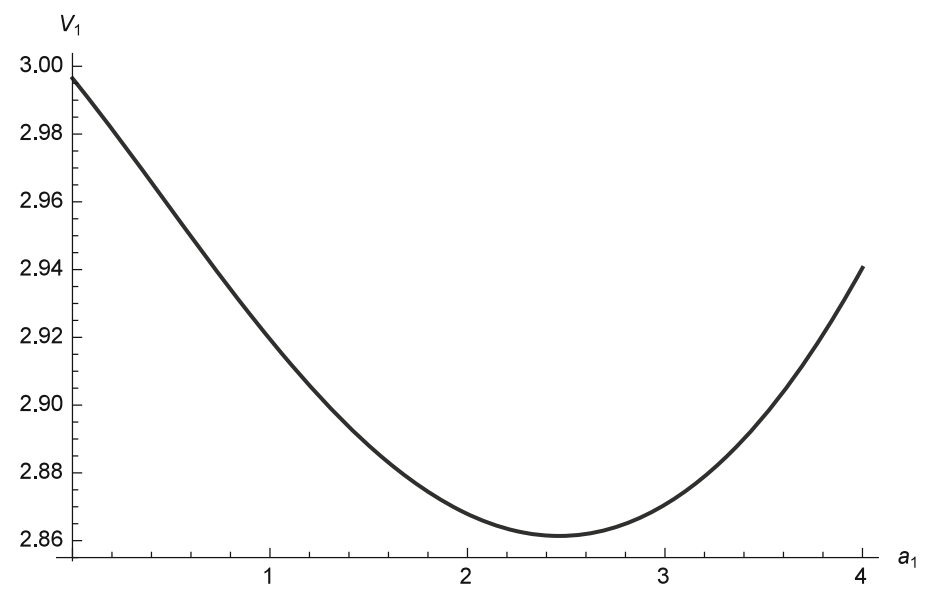

\section{Concluding remarks}

In this paper, we study the effects of altruism and spitefulness in a bilateral oligopoly. We prove that a trade equilibrium exists when agents have altruistic concerns for others on the opposite side of the market. The intuition behind this positive result is that

12 This can be verified with any computer algebra system. 
incentives to trade are strengthened under this configuration of altruistic concerns. By contrast, we show, by means of examples, that the non-trade equilibrium is the unique equilibrium in all other cases analyzed. These negative results are caused by the negativity of the necessary first-order conditions for optimality (as in Examples 1 and 2) and by the non-concavity of payoff functions (as in Example 3). Note that this negative results hold even when we consider bilateral oligopoly in which there are both altruistic and spiteful agents. For instance, one can consider a bilateral oligopoly with four agents where agents 1 and 2 are from Example 1 and agents 3 and 4 are from Example 2. In such bilateral oligopoly, the arguments on the first-order conditions used above apply, and therefore, the non-trade equilibrium is the unique equilibrium.

Before closing the paper, we wish to call attention to two points. First, we confine ourself to bilateral oligopolies with corner endowments in one commodity. We do not know whether our negative results extend to models with interior endowments. This is left for further research. Second, as in Dubey and Shubik (1985), one can show that the equilibrium of a bilateral oligopoly corresponds to the competitive equilibrium when there is a continuum of altruistic and spiteful agents. However, we still do not know whether the equilibrium of a bilateral oligopoly converges to the competitive equilibrium when the underlying exchange economy-with altruistic and spiteful agents-is replicated. The reason for this is that the standard convergence results do not apply (e.g., Lemma 4 of Dubey and Shubik 1978), even when there exists a trade equilibrium. This is a fruitful research area for future works.

Open Access This article is distributed under the terms of the Creative Commons Attribution 4.0 International License (http://creativecommons.org/licenses/by/4.0/), which permits unrestricted use, distribution, and reproduction in any medium, provided you give appropriate credit to the original author(s) and the source, provide a link to the Creative Commons license, and indicate if changes were made.

\section{A Appendix}

The proof of the existence theorem is based on three lemmas which require the following preliminary result.

Proposition 2 Let Assumption 2 hold. For eachagent $i \in I^{t}$, let $\gamma_{i}$ be such that $\gamma_{i}^{j}=0$ for each $j \in I^{t} \backslash\{i\}$ and $\gamma_{i}^{j} \geq 0$, for each $j \in I \backslash I^{t}$, for each $t=1$, 2. Then, for each $i \in I$, the utility function $V_{i}$ is continuous, strictly increasing and concave on $\mathbb{R}_{+}^{2}$, and continuously differentiable on $\mathbb{R}_{++}^{2}$.

Following Dubey and Shubik (1978), to prove the existence of a (Nash) equilibrium, we introduce a perturbed game $\Gamma^{\epsilon}(\gamma)$, with $\epsilon \in(0,1]$. This is a game defined as $\Gamma(\gamma)$ with the only exception that in the allocation rules (1) and (2) the ratio $\frac{B}{A}$ is replaced by $\frac{B+\epsilon}{A+\epsilon}$, i.e., the price of commodity $x$ becomes $\frac{B+\epsilon}{A+\epsilon}$. The interpretation is that an outside agency places a fixed offer of $\epsilon$ of both commodities. This does not change the strategy sets of agents, but does affect the prices, the final holdings, and the payoffs. We denote by $\left(\hat{a}^{\epsilon}, \hat{b}^{\epsilon}\right)$ an equilibrium of the perturbed game $\Gamma^{\epsilon}(\gamma)$.

In the first lemma, we prove the existence of an equilibrium in the perturbed game. 
Lemma 1 Let Assumptions 1-3 hold. For each $\epsilon \in(0,1]$, there exists an equilibrium for $\Gamma^{\epsilon}(\gamma)$.

Proof Consider an agent $i$ of type 1 and fix the strategies $\left(a_{-i}, b\right)$ for all other agents. In the perturbed game, the payoff function $V_{i}(x(a, b), y(a, b))$ is continuous, as $\frac{B+\epsilon}{A+\epsilon}$ is positive for each $\epsilon \in(0,1]$. Let

$$
\phi_{i}\left(a_{-i}, b\right) \in \underset{a_{i} \in S_{i}}{\operatorname{argmax}} V_{i}\left(x\left(\left(a_{i}, a_{-i}\right), b\right), y\left(\left(a_{i}, a_{-i}\right), b\right)\right)
$$

be the best response correspondence of the agent $i$. By the Weierstrass theorem, the best response correspondence $\phi_{i}$ is non-empty. We now show that the correspondence $\phi_{i}$ has convex-valued. Suppose that there are two feasible strategies $a_{i}^{\prime}$ and $a_{i}^{\prime \prime}$ which belong to $\phi_{i}\left(a_{-i}, b\right)$. We need to prove that $\tilde{a}_{i}=\delta a_{i}^{\prime}+(1-\delta) a_{i}^{\prime \prime}$, with $\delta \in(0,1)$, belongs to $\phi_{i}\left(a_{-i}, b\right)$. Since the strategies $\left(a_{-i}, b\right)$ are fixed, let us consider $(x(a, b), y(a, b))$ as functions of $a_{i}$, i.e., $\left(x\left(a_{i}\right), y\left(a_{i}\right)\right)$. Let $\left(x^{\prime}, y^{\prime}\right)=\left(x\left(a_{i}^{\prime}\right), y\left(a_{i}^{\prime}\right)\right)$, $\left(x^{\prime \prime}, y^{\prime \prime}\right)=\left(x\left(a_{i}^{\prime \prime}\right), y\left(a_{i}^{\prime \prime}\right)\right)$, and $(\tilde{x}, \tilde{y})=\delta\left(x^{\prime}, y^{\prime}\right)+(1-\delta)\left(x^{\prime \prime}, y^{\prime \prime}\right)$. Since the utility function $V_{i}$ is concave, by Proposition 2

$$
V_{i}(\tilde{x}, \tilde{y}) \geq \delta V_{i}\left(x^{\prime}, y^{\prime}\right)+(1-\delta) V_{i}\left(x^{\prime \prime}, y^{\prime \prime}\right)=V_{i}\left(x^{\prime}, y^{\prime}\right) .
$$

From the allocation rules (1) and (2), we have that $x_{i}\left(\tilde{a}_{i}\right)=\tilde{x}_{i}$, as $x_{i}\left(a_{i}\right)$ is linear; $y_{i}\left(\tilde{a}_{i}\right) \geq \tilde{y}_{i}$, as $y_{i}\left(a_{i}\right)$ is concave; $x_{j}\left(\tilde{a}_{i}\right)=\tilde{x}_{j}$, as $x_{j}\left(a_{i}\right)$ is linear, for each $j \in I^{2}$, and $y_{j}\left(\tilde{a}_{i}\right)=\tilde{y}_{j}$, as it does not depend on $a_{i}$, for each $j \in I^{2}$. But then

$$
V_{i}\left(x\left(\tilde{a}_{i}\right), y\left(\tilde{a}_{i}\right)\right) \geq V_{i}(\tilde{x}, \tilde{y})=V_{i}\left(x^{\prime}, y^{\prime}\right)=V_{i}\left(x\left(a^{\prime}\right), y\left(a^{\prime}\right)\right),
$$

as $V_{i}$ is strictly increasing by Proposition 2 . Thus, $\tilde{a}_{i}$ maximizes agent $i$ 's payoff function, and then, it belongs to $\phi_{i}\left(a_{-i}, b\right)$. Therefore, the correspondence $\phi_{i}$ has convex-valued. Furthermore, by the Berge Maximum theorem, $\phi_{i}$ is an upper hemicontinuous correspondence. If we consider an agent $i \in I^{2}$, then the previous argument leads, mutatis mutandis, to the same result and $\phi_{i}\left(a, b_{-i}\right)$ is a non-empty, convexvalued and upper hemicontinuous correspondence. As we are looking for a fixed point in the strategy space $S$, let us consider $\phi_{i}: S \rightarrow S_{i}$. Let $\Phi: S \rightarrow S$, such that $\Phi(S)=\prod_{i \in I} \phi_{i}(S)$. The correspondence $\Phi$ is non-empty, convex-valued, and upper hemicontinuous, because it is a product of non-empty, convex-valued, and upper hemicontinuous correspondences. Moreover, $S$ is a compact and convex set. Therefore, by the Kakutani fixed point theorem, there exists a fixed point $\left(\hat{a}^{\epsilon}, \hat{b}^{\epsilon}\right)$ of $\Phi$, which is an equilibrium of the perturbed game $\Gamma^{\epsilon}$.

In the next lemma, we prove that the price of commodity $x$ is finite and bounded away from zero at an equilibrium of any perturbed game.

Lemma 2 At an equilibrium of the perturbed game $\Gamma^{\epsilon}(\gamma),\left(\hat{a}^{\epsilon}, \hat{b}^{\epsilon}\right)$, there exist two positive constants $C$ and $D$, independent from $\epsilon$, such that

$$
C<\frac{\hat{B}^{\epsilon}+\epsilon}{\hat{A}^{\epsilon}+\epsilon}<D,
$$

for each $\epsilon \in(0,1]$. 
Proof It is straightforward to see that the proof provided by Dubey and Shubik (1978) still holds. To establish the existence of $C$, consider an agent $i$ of type 2. Following the same steps adopted by Dubey and Shubik (1978), we can apply the Uniform Monotonicity Lemma, as $u_{i}$ is continuous and strictly increasing, and we obtain the following relationship on internal utility functions (see p. 10 in Dubey and Shubik (1978)):

$$
u_{i}\left(x_{i}(\Delta), y_{i}(\Delta)\right)>u_{i}\left(x_{i}\left(\hat{a}^{\epsilon}, \hat{b}^{\epsilon}\right), y_{i}\left(\hat{a}^{\epsilon}, \hat{b}^{\epsilon}\right)\right) .
$$

The parameter $\Delta$ is a feasible increase in agent $i$ strategy and let $\left(x_{i}(\Delta), y_{i}(\Delta)\right)$ and $\left(x_{j}(\Delta), y_{j}(\Delta)\right)$, for each $j \in I^{1}$, be the new corresponding bundles. Note that

$$
\sum_{j \in I^{1}} u_{j}\left(x_{j}(\Delta), y_{j}(\Delta)\right)>\sum_{j \in I^{1}} u_{j}\left(x_{j}\left(\hat{a}^{\epsilon}, \hat{b}^{\epsilon}\right), y_{j}\left(\hat{a}^{\epsilon}, \hat{b}^{\epsilon}\right)\right)
$$

as $u_{j}$ is strictly increasing, by Assumption 2 , and $y_{j}(\Delta)>y_{j}\left(\hat{a}^{\epsilon}, \hat{b}^{\epsilon}\right)$, by allocation rule (1), for each $j \in I^{1}$. From the two previous inequalities, and since $\gamma_{i}^{j}=0$, for each $j \in I^{2} \backslash\{i\}$, and $\gamma_{i}^{j} \geq 0$, for each $j \in I^{1}$, we obtain that

$$
V_{i}(x(\Delta), y(\Delta))>V_{i}\left(x\left(\hat{a}^{\epsilon}, \hat{b}^{\epsilon}\right), y\left(\hat{a}^{\epsilon}, \hat{b}^{\epsilon}\right)\right) .
$$

Since $\left(\hat{a}^{\epsilon}, \hat{b}^{\epsilon}\right)$ is an equilibrium, we obtain the same contradiction of Dubey and Shubik (1978). By following their steps, we can then show that $\frac{\hat{B}^{\epsilon}+\epsilon}{\hat{A}^{\epsilon}+\epsilon}>C$. To establish the existence of $D$, consider an agent $i$ of type 1 . Then, the previous argument leads, mutatis mutandis, to $\frac{\hat{B}^{\epsilon}+\epsilon}{\hat{A}^{\epsilon}+\epsilon}<D$.

In the next lemma, we use the Kuhn-Tucker theorem to show that there exist two positive lower bounds for the sum of equilibrium offers $\hat{A}^{\epsilon}$ and $\hat{B}^{\epsilon}$ of the perturbed games. This lemma is crucial to prove that there exists a trade equilibrium for $\Gamma(\gamma)$.

Lemma 3 At an equilibrium of the perturbed game $\Gamma^{\epsilon}(\gamma),\left(\hat{a}^{\epsilon}, \hat{b}^{\epsilon}\right)$, there exist two positive constants $\alpha$ and $\beta$, independent from $\epsilon$, such that

$$
\alpha<\hat{A}^{\epsilon} \text { or } \beta<\hat{B}^{\epsilon}
$$

for each $\epsilon \in(0,1]$.

Proof Let $\left(\hat{a}^{\epsilon}, \hat{b}^{\epsilon}\right)$ be an equilibrium of the perturbed game $\Gamma^{\epsilon}(\gamma)$. We first consider the case in which there exists an agent $i \in I^{1}$ who satisfies Assumption 3. Then, $\hat{a}_{i}^{\epsilon}$ solves the following maximization problem:

$$
\begin{array}{ll}
\max _{a_{i}} & V_{i}\left(x\left(\left(a_{i}, \hat{a}_{-i}^{\epsilon}\right), \hat{b}^{\epsilon}\right), y\left(\left(a_{i}, \hat{a}_{-i}^{\epsilon}\right), \hat{b}^{\epsilon}\right)\right), \\
\text { subject to } & a_{i} \leq x_{i}^{0}, \\
& -a_{i} \leq 0 .
\end{array}
$$


By the Kuhn-Tucker theorem, there exist non-negative multipliers $\hat{\lambda}_{i}^{\epsilon}$ and $\hat{\mu}_{i}^{\epsilon}$, such that

$$
\begin{aligned}
& \left.\frac{\partial V_{i}}{\partial a_{i}}\right|_{\left(\hat{a}^{\epsilon}, \hat{b}^{\epsilon}\right)}-\hat{\lambda}_{i}^{\epsilon}+\hat{\mu}_{i}^{\epsilon}=0, \\
& \hat{\lambda}_{i}^{\epsilon}\left(\hat{a}_{i}^{\epsilon}-x_{i}^{0}\right)=0, \\
& \hat{\mu}_{i}^{\epsilon} \hat{a}_{i}^{\epsilon}=0 .
\end{aligned}
$$

By the assumption on the preference parameters, Eq. (6) can be written as

$$
\begin{gathered}
-\left.\frac{\partial u_{i}}{\partial x_{i}}\right|_{\left(\hat{a}^{\epsilon}, \hat{b}^{\epsilon}\right)}+\left.\frac{\partial u_{i}}{\partial y_{i}}\right|_{\left(\hat{a}^{\epsilon}, \hat{b}^{\epsilon}\right)} \frac{\hat{B}^{\epsilon}+\epsilon}{\hat{A}^{\epsilon}+\epsilon}\left(1-\frac{\hat{a}_{i}^{\epsilon}}{\hat{A}^{\epsilon}+\epsilon}\right) \\
+\left.\sum_{j \in I^{2}} \gamma_{i}^{j} \frac{\partial u_{j}}{\partial y_{j}}\right|_{\left(\hat{a}^{\epsilon}, \hat{b}^{\epsilon}\right)} \frac{\hat{b}_{j}^{\epsilon}}{\hat{B}^{\epsilon}+\epsilon}-\hat{\lambda}_{i}^{\epsilon}+\hat{\mu}_{i}^{\epsilon}=0 .
\end{gathered}
$$

Note that the summation over $j \in I^{2}$ is non-negative, as $u_{j}$ is strictly increasing, by Assumption 2, and $\gamma_{i}^{j} \geq 0$, by the assumption of the theorem, for each $j \in I^{2}$. Furthermore, the multiplier $\hat{\mu}_{i}^{\epsilon}$ is non-negative, by the Kuhn-Tucker theorem, and $\frac{\hat{B}^{\epsilon}+\epsilon}{\hat{A}^{\epsilon}+\epsilon}>C$, by Lemma 2 . But then, from the previous equation, we can derive the following inequality which must hold in equilibrium:

$$
-\left.\frac{\partial u_{i}}{\partial x_{i}}\right|_{\left(\hat{a}^{\epsilon}, \hat{b}^{\epsilon}\right)}+\left.\frac{\partial u_{i}}{\partial y_{i}}\right|_{\left(\hat{a}^{\epsilon}, \hat{b}^{\epsilon}\right)} C\left(\frac{\hat{A}^{\epsilon}-\hat{a}_{i}^{\epsilon}+\epsilon}{\hat{A}^{\epsilon}+\epsilon}\right)-\hat{\lambda}_{i}^{\epsilon}<0 .
$$

Now, fix the strategies $\left(\hat{a}_{-i}^{\epsilon}, \hat{b}^{\epsilon}\right)$ and suppose that $\hat{a}_{i}^{\epsilon} \rightarrow 0$. Then, we have that $\left.\frac{\partial u_{i}}{\partial y_{i}}\right|_{\left(\hat{a}^{\epsilon}, \hat{b}^{\epsilon}\right)} \rightarrow \infty$, as $\frac{d u_{i}^{2}(0)}{d y_{i}}=\infty$ by Assumption $3, \frac{\hat{A}^{\epsilon}-\hat{a}_{i}^{\epsilon}+\epsilon}{\hat{A}^{\epsilon}+\epsilon} \rightarrow 1$, and $\hat{\lambda}_{i}^{\epsilon}=0$, as constraint $(i)$ is not binding for sufficiently small $\hat{a}_{i}^{\epsilon}$. Moreover, $\frac{\partial u_{i}}{\partial x_{i}}$ is non-negative and has an upper bound, as $x_{i}\left(\hat{a}^{\epsilon}, \hat{b}^{\epsilon}\right) \rightarrow x_{i}^{0}>0$ and $\frac{\partial u_{i}}{\partial x_{i}}=\frac{d u_{i}^{1}}{d x_{i}}$ with $u_{i}^{1}$ being continuously differentiable for positive $x_{i}$ by Assumptions 2 and 3 . But then, there exists an $\alpha>0$, independent of $\epsilon$, such that the left-hand side of Eq. (7) is positive for each $\hat{a}_{i}^{\epsilon} \in[0, \alpha]$. Hence, since the inequality (7) must hold in equilibrium, $\hat{a}_{i}^{\epsilon}>\alpha$, and, a fortiori, $0<\alpha<\hat{A}^{\epsilon}$, for each $\epsilon \in(0,1]$. We now consider the case in which there exists an agent $i \in I^{2}$ who satisfies Assumption 3. Then, the previous argument leads, mutatis mutandis, to $0<\beta<\hat{B}^{\epsilon}$, for each $\epsilon \in(0,1]$.

We can now prove the existence theorem.

Proof Consider a sequence of $\left\{\epsilon_{n}\right\}_{n}$ converging to 0 . By Lemma 1, in each perturbed game, there exists an equilibrium. Then, we can consider a sequence of equilibria $\left\{\left(\hat{a}^{\epsilon_{n}}, \hat{b}^{\epsilon_{n}}\right)\right\}_{n}$. Since $S$ is compact and $\frac{\hat{B}^{\epsilon_{n}}+\epsilon_{n}}{\hat{A}^{\epsilon_{n}}+\epsilon_{n}} \in[C, D]$, by Lemma 2, we can pick a subsequence of $\left\{\left(\hat{a}^{\epsilon_{n}}, \hat{b}^{\epsilon_{n}}\right)\right\}_{n}$ that converge to $(\hat{a}, \hat{b})$ such that $(\hat{a}, \hat{b}) \in S$ and $\frac{\hat{B}}{\hat{A}} \in$ 
$[C, D]$. But then, the strategy profile $(\hat{a}, \hat{b})$ is a point of continuity of payoff functions, and then, it is an equilibrium of $\Gamma(\gamma)$. The result of Lemma 3 implies that $\hat{A}>0$ or $\hat{B}>0$. But then, since $\frac{\hat{B}}{\hat{A}} \in[C, D]$, we can conclude that $\hat{A}>0$ and $\hat{B}>0$. Hence, $(\hat{a}, \hat{b})$ is a trade equilibrium.

We finally prove Proposition 1.

Proof Assume that there exists a trade equilibrium $(\hat{a}, \hat{b})$. Then, $\hat{a}_{i}>0$ for at least two agents $i$ of type 1 (see footnote 8). Consider the payoff maximization problem for the agent $i$ of type 1 , such that $\hat{a}_{i}>0$ [see (5)]. By the Kuhn-Tucker theorem and the assumption on the preference parameters, the necessary first-order condition for optimality of agent $i$ can be written as

$$
\begin{gathered}
\left.\frac{\partial V_{i}}{\partial a_{i}}\right|_{(\hat{a}, \hat{b})}=-\left.\frac{\partial u_{i}}{\partial x_{i}}\right|_{(\hat{a}, \hat{b})}+\left.\frac{\partial u_{i}}{\partial y_{i}}\right|_{(\hat{a}, \hat{b})} \frac{\hat{B}}{\hat{A}^{2}}\left(\hat{A}-\hat{a}_{i}\right) \\
+\left.\sum_{j \in I^{1} \backslash\{i\}} \gamma_{i}^{j} \frac{\partial u_{j}}{\partial y_{j}}\right|_{(\hat{a}, \hat{b})}\left(-\hat{a}_{j} \frac{\hat{B}}{\hat{A}^{2}}\right)-\hat{\lambda}_{i}+\hat{\mu}_{i}=0,
\end{gathered}
$$

with $\hat{\mu}_{i}=0$, as $\hat{a}_{i}>0$, and $\hat{\lambda}_{i} \geq 0$, by the Kuhn-Tucker theorem. From the previous equation, we can derive the following inequality which holds at any trade equilibrium

$$
-\left.\frac{\partial u_{i}}{\partial x_{i}}\right|_{(\hat{a}, \hat{b})}+\frac{\hat{B}}{\hat{A}^{2}} \sum_{j \in I^{1} \backslash\{i\}} \hat{a}_{j}\left(\left.\frac{\partial u_{i}}{\partial y_{i}}\right|_{(\hat{a}, \hat{b})}-\left.\gamma_{i}^{j} \frac{\partial u_{j}}{\partial y_{j}}\right|_{(\hat{a}, \hat{b})}\right) \geq 0 .
$$

We now prove the statement of the proposition by contradiction and we suppose that (3) does not hold for each $i, j \in I^{1}$, with $i \neq j$. This implies that the summation in (8) is negative. Since $\frac{\partial u_{i}}{\partial x_{i}} \geq 0$ as the utility function is strictly increasing by Assumption 2, then the previous inequality does not hold, a contradiction.

\section{References}

Amir, R., Bloch, F.: Comparative statics in a simple class of strategic market games. Games Econ. Behav. 65, 7-24 (2009)

Bergstrom, T.: Puzzles: love and spaghetti, the opportunity cost of virtue. J. Econ. Perspect. 3, 165-173 (1989)

Bloch, F., Ghosal, S.: Stable trading structures in bilateral oligopolies. J. Econ. Theory 74, 368-384 (1997) Bloch, F., Ferrer, H.: Trade fragmentation and coordination in strategic market games. J. Econ. Theory 101, 301-316 (2001)

Bourlès, R., Bramoullé, Y., Perez-Richet, E.: Altruism in networks. Econometrica 85, 675-689 (2017)

Dickson, A., Hartley, R.: The strategic Marshallian cross. Games Econ. Behav. 64, 514-532 (2008)

Dubey, P., Shubik, M.: The noncooperative equilibria of a closed trading economy with market supply and bidding strategies. J. Econ. Theory 17, 1-20 (1978)

Dubey, P., Shubik, M.: Perfect competition in strategic market games with interlinked preferences. Econ. Lett. 17, 3-4 (1985)

Dufwenberg, M., Heidhues, P., Kirchsteiger, G., Riedel, F., Sobel, J.: Other-regarding preferences in general equilibrium. Rev. Econ. Stud. 78, 613-639 (2011) 
Edgeworth, F.Y.: Mathematical Psychics. Augustus M. Kelley., New York (1881). (new editions 1888, 1932, 1967, 1981)

Fehr, E., Gachter, S.: Fairness and retaliation: the economics of reciprocity. J. Econ. Perspect. 14, 159-181 (2000)

Gabszewicz, J., Michel, P.: Oligopoly equilibrium in exchange economies. In: Eaton, B., Harris, R. (eds.) Trade, Technology and Economics: Essays in Honor of Richard G Lipsey, pp. 217-240. Elgar, Cheltenham (1997)

Graziano, M.G., Meo, C., Yannelis, N.C.: Stable sets for exchange economies with interdependent preferences. J. Econ. Behav. Organ. 140, 267-286 (2017)

Kreps, D.: Microeconomic Foundations 1: Choice and Competitive Markets. Princeton University Press, Princeton (2012)

Levine, D.K.: Modelling altruism and spitefulness in experiments. Rev. Econ. Dyn. 1, 593-622 (1998)

Shapley, L., Shubik, M.: Trade using one commodity as a means of payment. J. Polit. Econ. 85, 937-968 (1977)

Sobel, J.: Interdependent preferences and reciprocity. J. Econ. Lit. 43, 396-440 (2005)

Sobel, J.: Markets and Other-Regarding Preferences. Discussion Paper, Economics Department. University of California, San Diego (2010)

Ythier, J.M.: The economic theory of gift-giving: perfect substitutability of transfers and redistribution of wealth. In: Kolm, S.C., Ythier, J.M. (eds.) Handbook of the Economics of Giving, Altruism and Reciprocity. Elsevier, Amsterdam (2006)

Publisher's Note Springer Nature remains neutral with regard to jurisdictional claims in published maps and institutional affiliations. 